\title{
Infrared conductivity of metallic (III,Mn)V ferromagnets
}

\author{
Jairo Sinova $^{1}$, T. Jungwirth ${ }^{1,2}$, S.- R. Eric Yang ${ }^{1,3}$, J. Kučera ${ }^{2}$, and A.H. MacDonald ${ }^{1}$ \\ ${ }^{1}$ Department of Physics, The University of Texas at Austin, Austin, TX 78712 \\ ${ }^{2}$ Institute of Physics ASCR, Cukrovarnická 10, 162 53 Praha 6, Czech Republic \\ ${ }^{3}$ Department of Physics, Korea University, Seoul 136-701, Korea
}

(November 13, 2018)

\begin{abstract}
We present a theory of the infrared conductivity and absorption coefficients of metallic (III,Mn) V ferromagnetic semiconductors. We find that the conductivity is dominated by inter-valence-band transitions that produce peaks at $\hbar \omega \sim 220 \mathrm{meV}$ and obscure the broadened Drude peak. We demonstrate that transverse f-sum rule measurements can be used to extract accurate values for the free carrier density, bypassing the severe characterization difficulties that have till now been created by the large anomalous Hall effect in these materials.
\end{abstract}

Following the discovery of carrier-induced ferromagnetism in (In,Mn)As [1] and (Ga,Mn)As [2] in the early nineties, studies of ferromagnetism in (III,Mn)V diluted magnetic semiconductors (DMS's) have yielded many surprises. The transport and optical properties of these heavily-doped semiconductors are richer than those of conventional itinerant electron ferromagnets because of strong valence-band spin-orbit coupling, and because of the sensitivity of their magnetic state to growth conditions, doping, and external fields. These novel ferromagnets are likely to have a major technological impact [3] if systems with Curie temperatures above room temperature can be created and better control of disorder effects can be achieved. We present a theory of the infrared conductivity of (III,Mn)V ferromagnets which we believe will increase the value of this powerful probe in characterizing present and future DMS ferromagnets. In particular, we demonstrate that infrared conductivity measurements in metallic samples can be used to obtain accurate measurements of the total free carrier density, likely the key to understanding the now firmly established [4 dependence of magnetic properties on growth and post-growth annealing conditions. This experimental possibility is especially important in these semiconductors because they are ferromagnetic and have a large anomalous Hall conductivity [5] which severely complicates Hall-effect carrier-density measurements, [2,6] even when they are performed in very strong magnetic fields.

Our conclusions are based on a semi-phenomenological model [7 10] in which host semiconductor valence band electrons, described by a k.p model, interact with randomly distributed aligned $(T=0)$ Mn local- moment acceptors via Coulomb and short-range exchange interactions, and via Coulomb interactions only with the compensating charged defects known to be present. Some of our considerations are based on standard linear-response theory expressions for the ac conductivity of weakly disordered metals, in which disorder is included only through finite quasiparticle lifetimes and localization ef- fects are ignored. In the dc limit, with quasiparticle scattering rates evaluated using Born approximation expressions for scattering off the spatial fluctuations of the screened-Coulomb and exchange potentials, these expressions imply mobilities [11] that are consistent with values measured in optimally annealed, high- $T_{c}$, DMS ferromagnets. Although the predictions discussed below are intended to be most reliable for the most metallic systems, they appear to explain much currently available infrared optical data, most of which has been obtained in studies of systems with relatively low dc conductivities.

The main features observed in experiments [12 15] are the absence of a clear low-frequency Drude peak, and a broad absorption peak near $220 \mathrm{meV}$ that becomes stronger as the samples are cooled. The $220 \mathrm{meV}$ peak has been attributed either to inter-valence-band transitions, to transitions between the semiconductor valence band states and a Mn induced impurity band, or to a combination of these contributions. Dynamical meanfield-theory studies [16], for a single-band model that neglects the spin-orbit coupling and the heavy-light degeneracy of a III-V semiconductor valence band, demonstrate that non-Drude impurity-band related features can occur in DMS ferromagnets. The conductivities predicted by this model are, however, inconsistent with experiment in magnitude and in temperature trend. As demonstrated in the following paragraphs, realistic features of the valence band electronic structure are key to understanding the conductivity data. We find that the absorption peak near $220 \mathrm{meV}$ is associated with heavyhole to light-hole inter-valence-band transitions, and that transitions to the bands split-off by spin-orbit interactions are responsible for the gradual increase in absorption observed in experiments [13] at frequencies above $500 \mathrm{meV}$.

The physical picture we have developed for the infrared conductivity starts from an examination of the limit in which disorder is ignored. In building a picture starting from this limit we are motivated in part by the observa- 
tion that many magnetic and transport property trends (critical temperatures, magnetic anisotropy and its strain dependence, magnetic stiffness, anomalous Hall effect, etc. 17 10,17, 18) in DMS ferromagnets appear to be adequately described without accounting for disorder, even though it is relatively strong by some measures. The linear response of the system to a light probe is characterized by the optical conductivity tensor $\sigma_{\alpha, \beta}(\omega)$. In thin film absorption measurements, the real part of the conductivity is related to the absorption coefficient by

$$
\tilde{\alpha}(\omega)=2 \frac{\operatorname{Re}[\sigma(\omega)]}{Y+Y_{0}},
$$

where $Y$ and $Y_{0}$ are the admittances of the substrate and free space, respectively [19]. We have evaluated $\sigma_{\alpha, \beta}(\omega)$ at $T=0$ using the standard Kubo formula for non-interacting quasi-particles [20]. The clean-limit ac conductivity of our model is plotted in Fig. 1 for a typical set of $(\mathrm{Ga}, \mathrm{Mn})$ As parameter values, carrier density $p=0.3 \mathrm{~nm}^{-3}, 5 \% \mathrm{Mn}$ substitution, implying a Mn concentration $N_{M n}=1.1 \mathrm{~nm}^{-3}$, and band parameters that incorporate the strain produced by epitaxial growth on a GaAs substrate [21]. When disorder is neglected, Coulomb interactions have no effect, while the exchange interactions produce a uniform exchange mean Zeeman field, $\vec{h}=J_{p d} N_{M n} S \hat{\Omega}$, where $\hat{\Omega}$ is the magnetization direction. The finite-frequency conductivity results from transitions between occupied and unoccupied valence bands states; the band Kramers degeneracies are split by an amount comparable to the Fermi energy. $\left(J_{p d}=55 \mathrm{meV} \mathrm{nm}^{3}\right.$ is the exchange-coupling constant so that $h \sim 150 \mathrm{meV}$.) The results illustrated in Fig. 1 are for $\hat{\Omega}$ along the growth $(\langle 001\rangle)$ direction, the magnetic easy-axis for a tensile strained (Ga,Mn)As layers [9]; clean limit results for samples under compressive strain (GaAs substrates) which have in plane easy axes differ in detail [21. The two split-peaks present in the clean limit at $\sim 250$ and $\sim 325 \mathrm{meV}$ correspond to heavy-hole to light-hole transitions and to light-hole to split off (s-o) band transitions, respectively. Beyond $\sim 500 \mathrm{meV}$ there is a slight rise in the absorption which corresponds to the heavy-hole to s-o split off band transition. In the clean limit, the conductivity has a $\delta$-function contribution at $\omega=0$ due to intraband transitions. Its relative contribution to the f-sum rule of our model, discussed further below, is $35 \%$, nearly independent of carrier density over the relevant range.

We account for disorder by including the lifetime broadening of quasiparticle spectral functions in evaluating the Kubo formula. The effective lifetime for transitions between bands $n$ and $n^{\prime}, \tau_{n, n^{\prime}}$, is calculated by averaging quasiparticle scattering rates calculated from Fermi's golden rule including both screened Coulomb and exchange interactions [11]. The solid curve in Fig. 11 illustrates the influence of disorder on the conductivity. The intraband conductivity evolves into a broadened Drude peak that overlaps with broadened interband absorption features. In Fig. 2, we plot ac conductivities calculated in this way for Ga.95 Mn ${ }_{.05}$ As at a series of carrier densities. In our model, an increase in carrier density is accompanied by a decrease in the density $N_{c}$ of compensating charged defects; we assume that all have charge $Z=2$ so that $p=N_{M n}-2 N_{c}$ [22]. As $p$ increases, $N_{c}$ and quasiparticle scattering rates decrease in tandem. The evaluation of quasiparticle scattering rates allows us to check the consistency of our weak-scattering approach, which requires $\hbar /\left(\tau_{n n^{\prime}} E_{F}\right)>1$, where $E_{F}$ is the hole Fermi energy. For $5 \% \mathrm{Mn}$ doping, the condition is safely satisfied only for $p \gtrsim 0.3 \mathrm{~nm}^{-3}$. As the carrier density increases above this value we see in Fig. 2 that the peak near $220 \mathrm{meV}$ sharpens and the low frequency Drude peak emerges more clearly.

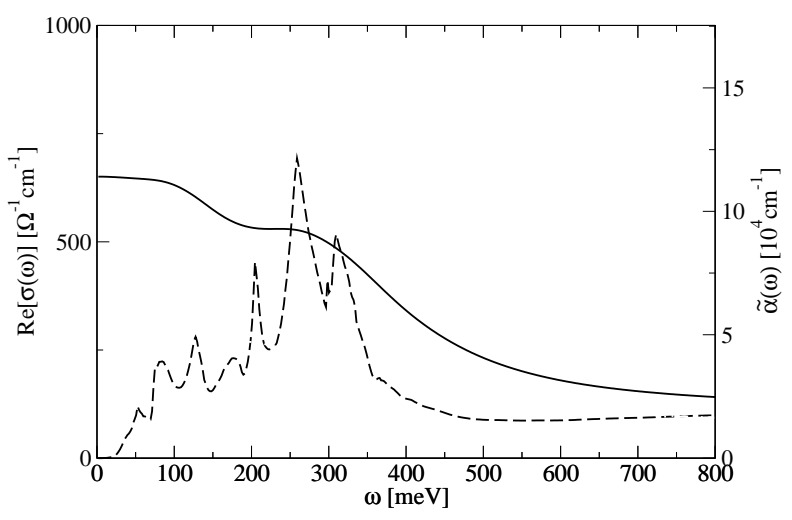

FIG. 1. Optical conductivity $\operatorname{Re}[\sigma(\omega)]$ and absorption coefficient $\tilde{\alpha}(\omega)$ for carrier density $n=0.3 \mathrm{~nm}^{-3}$ in $\mathrm{Ga}_{0.95} \mathrm{Mn}_{0.05} \mathrm{As}$ without (dashed) and with (solid) impurity scattering present.

Since all model parameters are accurately known from other experiments, the ac conductivity values in Fig. 2 are parameter free. Although direct comparisons with infrared conductivity experiments is difficult because the carrier density is not usually known, our dc conductivities are generally larger than those of optimally annealed samples [4]. Our quasiparticle scattering rates are likely smaller than those in any current experimental sample, due to some combination of inaccuracy in our approximate scattering amplitudes and unknown sources of additional unintended disorder. Further progress in sorting out variable transport and magnetic properties requires accurate experimentally determined carrier density values, which we believe can be obtained directly from infrared conductivity data as we explain below.

In the following paragraphs we discuss the f-sum rule of our model: 


$$
F \equiv \int_{0}^{\infty} d \omega \operatorname{Re}\left[\sigma_{x x}(\omega)\right]=\frac{\pi e^{2}}{2 V} \sum_{\alpha} f_{\alpha}\left\langle\alpha\left|\frac{\partial^{2} H_{b a n d}}{\hbar^{2} \partial k_{x}^{2}}\right| \alpha\right\rangle .
$$

In this equation $f_{\alpha}$ is a quasiparticle Fermi factor and $\partial^{2} H_{\text {band }} / \hbar^{2} \partial k_{x}^{2}$ is the $x x$ component of the k.p model inverse effective mass operator, which is diagonal in envelope function wavevector but off diagonal in envelope function spinor index. We emphasize that this sum rule is completely independent of the weak-scattering approximations on which the ac conductivity calculations discussed above were based. In practical applications, it is necessary to choose an upper cut-off for the frequency integral [23]. We define a weakly cut-off dependent optical effective mass for DMS ferromagnets by defining $F=\pi e^{2} p / 2 m_{\text {opt }}$. As illustrated in the inset of Fig 2, for the case of cut-off frequency $\hbar \omega_{\max }=800 \mathrm{meV}$, the $\mathrm{f}$ sum rule values of $F$ evaluated from our weak scattering theory are accurately linear in $p$ over the entire range of relevant carrier densities. The optical masses evaluated here, which provide the f-sum rule with practical utility, represent a complicated average over heavy and light hole masses in the spin-split ferromagnetic valence bands. Disorder does have a small but measurable effect on $m_{\text {opt }}$ as illustrated in Fig. 2. By solving self-consistent meanfield calculations for finite-size realizations of our DMS ferromagnet model, we have established that a fully nonperturbative treatment of disorder alters $m_{\text {opt }}$, by less than $10 \%$ for metallic DMS ferromagnets 24,25]. We have also verified that the optical mass is not perceptibly dependent on the magnetization orientation and that it is altered by less than $1 \%$ by the exchange mean-field. Using the six-band Luttinger model, the optical masses for GaAs, InAs, and GaSb DMS ferromagnets with a 800,400 , and $700 \mathrm{meV}$ cutoffs are $0.25-0.29 \mathrm{~m}_{e}, 0.40-0.43$ $\mathrm{m}_{e}$, and $0.21-0.23 \mathrm{~m}_{e}$ respectively, the extremes of these ranges corresponding to the clean (lower) and disordered (upper) limits of our model [26]. $m_{\text {opt }}$ depends to a good approximation only on the frequency cutoff and on the Luttinger parameters that characterize the host semiconductor valence bands [27, permitting accurate carrierdensity measurements based on the f-sum rule.

We have applied our procedure for determining the DMS carrier density to a sample with $4 \% \mathrm{Mn}$ doping, measured by Katsumoto et al [13], obtaining a value of $0.17 \pm .03 \mathrm{~nm}^{-3}$ (Their data is shown as the dot-dashed line in Fig. 2). Although their sample is strongly disordered, the f-sum rule based density estimate still appears reasonable. It is consistent, within $30 \%$, with the [21]28] carrier density that would produce a ferromagnetic state with the measured [13] critical temperature of $90 \mathrm{~K}$. Note that the hole density estimate [13] based on the measured room temperature Hall coefficient for the same sample is $\sim 5 \times$ smaller than that expected on the basis of its critical temperature, emphasizing once again the difficulty of extracting carrier densities from Hall data.

The strong spin-orbit coupling in the (Ga,Mn)As valence band $\left(\Delta_{s o}=341 \mathrm{meV}\right)$ compared to the typical spectral broadening of the hole quasiparticles $(\hbar / \tau \sim$ $100-200 \mathrm{meV}$ ) leads to a dependence of the absorption spectrum on the direction of the magnetization. This dependence should lead to measurable infrared conductivity changes that can be induced by an external magnetic field larger than the sample's magnetocrystalline anisotropy field $\sim 100 \mathrm{mT}[8]$, applied along the hard $\langle 100\rangle$ magnetic axis. The inset of Fig. 3 shows the orientation dependence of the optical conductivity and absorption coefficients for the same sample parameters as in Fig. 1, calculated neglecting disorder and with the incident light polarization along the $\langle 100\rangle$ direction. In the main plot of Fig. 3 we compare absorption spectra evaluated using our weak disorder approximation. The sharp spectral features near $325 \mathrm{meV}$ are smeared out by disorder, however, the $\sim 15 \mathrm{meV}$ relative shift of the absorption peak for the two magnetization orientations is nearly disorder independent and should be observable experimentally. This effect is stronger when the density of compensating defects is reduced.

In summary, we find that the infrared conductivity of metallic DMS ferromagnets has Drude and interband contributions that have approximately equal importance. In current samples these contributions overlap in frequency, but we expect that they will begin to separate if recent improvements in sample conductivity can be extended. We predict that the infrared conductivity will be very sensitive to changes in compensating defect and carrier densities, believed to be responsible for the sensitivity of DMS ferromagnets to growth and annealing conditions. In particular, we have evaluated optical effective masses that will allow carrier densities to be extracted from f-sum rule measurements, circumventing difficulties posed by the large anomalous Hall effect of (III,Mn)V ferromagnetic semiconductors. Simultaneous measurement of optical, magnetic, and dc transport properties should prove to be potent in advancing the materials science of these interesting systems, when our optical masses are used to extract carrier densities. We have also demonstrated the sensitivity of the infrared optical conductivity to magnetization orientation that is due to the strong spin-orbit coupling in these systems.

We acknowledge helpful discussions with Jacek Furdyna, Bruce McCombe, Andrea Markelz, Joaquin Fernandez-Rossier, Peter Schiffer, and Jason Singley. The work was supported by the Welch Foundation, DARPA, the Grant Agency of the Czech Republic under grant 202/02/0912, the Ministry of Education of the Czech Republic under grant OC P5.10, and KOSEF Quantum-functional Semiconductor Research Center at Dongguk University. 
[1] H. Ohno, H. Munekata, T. Penney, S. von Molnár, and L.L. Chang, Phys. Rev. Lett. 68, 2664 (1992).

[2] H. Ohno, A. Shen, F. Matsukura, A. Oiwa, A. Endo, S. Katsumoto, and Y. Iye, Appl. Phys. Lett. 69, 363 (1996).

[3] T. Dietl, Acta Phys. Polon. A 100, 139 (2001).

[4] S. J. Potashnik, et al, App. Phys. Lett., 79, 1495 (2001) and S. J. Potashnik, et al cond-mat/0204250.

[5] T. Jungwirth, Q. Niu, and A.H. MacDonald, e-print http://arXiv.org/abs/cond-mat/0110484.

[6] H. Ohno, J. Magn. Magn. Mater. 200, 110 (1999).

[7] T. Dietl, H. Ohno, F. Matsukura, J. Cibert, and D. Ferrand, Science 287, 1019 (2000).

[8] T. Dietl, H. Ohno, and F. Matsukura, Phys. Rev. B 63, 195205 (2001).

[9] M. Abolfath, T. Jungwirth, J. Brum, and A.H. MacDonald, Phys. Rev. B 63, 054418 (2001).

[10] J. König, J. Schliemann, T. Jungwirth, and A.H. MacDonald, in Electronic Structure and Magnetism of Complex Materials, edited by D.J. Singh and D.A. Papaconstantopoulos (Springer Verlag 2002), e-print http://arXiv.org/abs/cond-mat/0111314.

[11] M. Abolfath, T. Jungwirth, J. Sinova, J. Kučera, and A.H. MacDonald, unpublished.

[12] Y. Nagai et al., Jpn. J. Appl. Phys. 40, 6231 (2001).

[13] S. Katsumoto et al., Mat. Sci. Eng. B 84, 88 (2001).

[14] K. Hirakawa et al., Phys. Rev. B 65, 193312 (2002).

[15] E.J. Singley, D.N. Basov, R.K. Kawakami, D.D. Awschalom, Bull. Am. Phys. Soc., [2002 March meeting, Abstract G19.001]

[16] E.H. Hwang, A.J. Millis, and S. Das Sarma, condmat/0202071

[17] T. Dietl, A. Haury, and Y. Merle d'Aubigné, Phys. Rev. B 55, R3347 (1997).

[18] T. Jungwirth, W.A. Atkinson, B.H. Lee, and A.H. MacDonald, Phys. Rev. B 59, 9818 (1999).

[19] This formula is applicable in the far infrared regime. At higher frequencies in the spectrum multiple scattering from the interfaces have to be taken into account. This can be easily done using the standard electromagnetic formalism of light waves propagating through a medium. Note that $\tilde{\alpha}(\omega)$ is not the same as the bulk absorption coefficient usually defined as $\alpha(\omega)=2 \omega \operatorname{Im}[\sqrt{\epsilon(\omega)}] / c$.

[20] See for example F. Wooten, Optical Properties of Solids, Academic Press, New York (1972).

[21] A large database with our theory predictions for different sample parameters can be accesses via http://unix12.fzu.cz/ms/index.php

[22] There is a broad consensus that variation in the density of compensating defects, believed to consist mainly of As antisites and $\mathrm{Mn}$ interstitials, is responsible for most of the sensitivity of DMS ferromagnets to growth and annealing conditions. See for example K. M. Yu, W. Walukiewicz, T. Wojtowicz, I. Kuryliszyn, X. Liu, Y. Sasaki, and J. K. Furdyna, Phys. Rev. B., Rapid Comm., in press (2002).

[23] Note that Eq. 2, is useful only when the maximum fre- quency of the intra-valence-band infrared conductivity is well below the sum of the semiconductor gap and the Fermi energy. This conditions is reasonably well satisfied for most DMS ferromagnets. Note also that the application of a frequency cutoff to such equation necessarily increases the $m_{o} p t$ measured.

[24] As the carrier density drops below $\sim 0.2 \mathrm{~nm}^{-3}$, disorder localizes the valence band electrons, S.-R. Eric Yang and A.H. MacDonald, preprint cond-mat/0202021. More strongly localized valence band holes have more heavyhole character, causing the the optical mass to increase in comparison to values that are appropriate in the metallic limit.

[25] S.-R. Eric Yang, Y.P. Shim, J. Sinova, T. Jungwirth, and A.H. MacDonald, Bull. Am. Phys. Soc. [2002 March Meeting, Abstract G19.002].

[26] For comparison, the result for GaAs for the full sum rule gives $m_{o} p t=0.239$ independent of disorder.

[27] I. Vurgaftman, J.R. Meyer, and L.R. Ram-Mohan, J. Appl. Phys 89, 5815 (2001).

[28] T. Jungwirth, J. König, J. Sinova, J. Kucera, and A.H. MacDonald, Phys. Rev. B, Rapid Comm., in press.

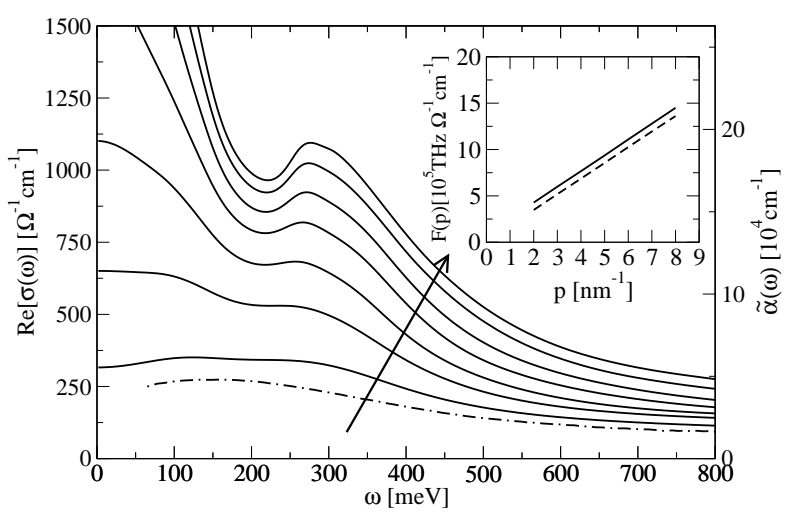

FIG. 2. Optical conductivity $\operatorname{Re}[\sigma(\omega)]$ and absorption coefficient $\tilde{\alpha}(\omega)$ for carrier densities from $p=0.2$ to $0.8 \mathrm{~nm}^{-3}$ in the direction indicated by the arrow, for $\mathrm{Ga}_{0.95} \mathrm{Mn}_{0.05} \mathrm{As}$. The dot-dashed line is an experimental absorption curve for a sample with a Mn concentration of $4 \%$ obtained by Hirakawa et al. The inset shows the spectral weight $F(p)$ evaluated with a frequency cut-off of $800 \mathrm{meV}$ including disorder (dashed) and in the clean limit (solid). 


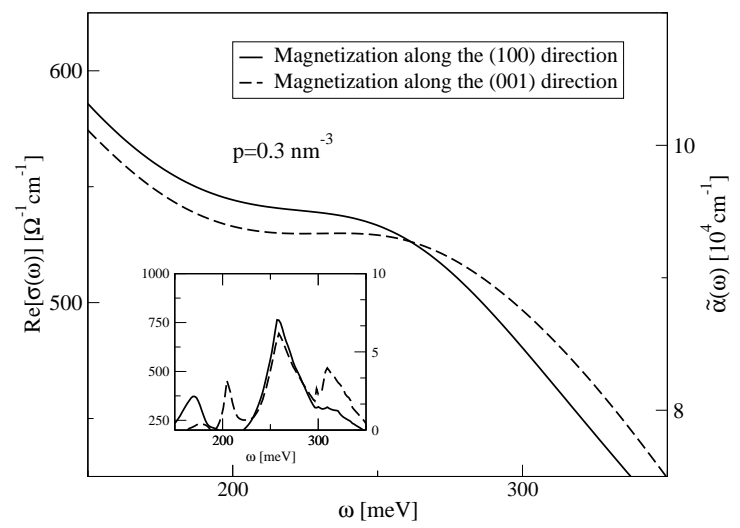

FIG. 3. Magnetization orientation dependence of the optical conductivity $\operatorname{Re}[\sigma(\omega)]$ and absorption coefficient $\tilde{\alpha}(\omega)$ for carrier densities $n=0.3 \mathrm{~nm}^{-3}$ for $\mathrm{Ga}_{0.95} \mathrm{Mn}_{0.05} \mathrm{As}$ at $T=0$. The light is linearly polarized along the $\mathrm{x}$-direction. The inset indicates the results for the disorder free case. 